\title{
Estimating Benefits of Deep Retrofit Scenarios Based on Passivhaus Standards in the Residential Sector of Waterloo Region
}

\author{
Qi Wang ${ }^{1,2, *}$, Paul Parker ${ }^{2}$ \\ ${ }^{1}$ Department of Water Resources and Environment, China University of Geosciences, China \\ ${ }^{2}$ Department of Geography and Environmental Management, University of Waterloo, Canada
}

\begin{abstract}
This paper investigates the potential benefits of deep retrofit scenarios on the energy consumption of houses in Waterloo Region. The building envelop is the primary focus, especially upgrading wall, attic, and foundation insulation. The sample houses are divided into six categories based on their construction date. A deep retrofit upgrade scenario is applied to a typical house of each category and the potential energy savings and $\mathrm{CO}_{2}$ emissions reduction of each typical house are estimated after completing the corresponding retrofit scenario. One of the six retrofitted houses achieves the Passive House energy intensity standard. Finally, the total potential savings for Waterloo Region are obtained based on the Regional housing census. According to the above analysis, it can be concluded that applying deep retrofit scenarios to in the residential sector of Waterloo Region can make significant contributions to reducing $14.16 \times 10^{9} \mathrm{MJ} / \mathrm{yr}$ energy consumption and $7.83 \times 10^{8} \mathrm{~kg} / \mathrm{yr} \mathrm{CO}_{2}$ emissions.
\end{abstract}

\section{Introduction}

In Canada, the residential sector is the third largest energy consumer, accounting for $17 \%$ of the total national energy consumption and $15 \%$ of greenhouse gas emissions [1]. In Waterloo Region, the residential and business activities emitted approximately 3.6 million tonnes of greenhouse gases in 2010, and the residential sector accounted for $22 \%$ of the total [2]. This situation prompts us to examine the potential impacts of increasing energy efficiency and reducing energy consumption in homes. Residential retrofit scenarios to reduce energy consumption are developed for Waterloo Region [3].

The objective of this research is to evaluate scenarios to improve residential energy performance and reduce $\mathrm{CO}_{2}$ emissions by applying retrofit scenarios based on Passivhaus Standards to houses [4] in Waterloo Region.

The data employed in this study are derived from a standard residential energy computer model (Hot2XP), prepared as part of the residential energy efficiency project (REEP) [5] and data tables from Statistics Canada [6]. The sample houses were divided into six categories according to their construction date, and then a typical house from each category was selected based on the similarities between its energy consumption characteristics and the corresponding category. Retrofit scenarios are applied to the six typical houses and then the energy savings and $\mathrm{CO}_{2}$ emissions reduction of these six typical houses are estimated. The total potential energy savings and $\mathrm{CO}_{2}$ emissions reduction of Waterloo Region can be obtained based on the Waterloo Region housing census and the number of houses in each age category.

\section{Methodology}

In this study, the sample houses are divided into six categories based on the age classification of occupied private dwellings (Table 1).
Table 1. Classification of occupied private dwellings

\begin{tabular}{|c|c|c|c|c|c|c|}
\hline Classification & C1 & C2 & C3 & C4 & C5 & C6 \\
\hline Construction & Before & $1946-$ & $1961-$ & $1971-$ & $1981-$ & $1991-$ \\
Period & 1946 & 1960 & 1970 & 1980 & 1990 & 2000 \\
\hline
\end{tabular}

Typical houses were selected based on the similarities between their energy consumption characteristics and the average values of the corresponding category. Key energy characteristics include the total energy consumption, the amount of heat loss through air leakage, foundation floor / walls, attic, main walls, and windows / doors. The six selected houses, were constructed in 1945, 1959, 1966, 1975, 1986, and 1995, respectively.

The comparison of current $\mathrm{R}$ values of segments of the six typical houses to the deep retrofit $\mathrm{R}$ values is presented in Table 2.

Table 2. Comparison of current and deep retrofit $\mathrm{R}$ values

\begin{tabular}{|c|c|c|c|c|c|c|c|}
\hline \multirow{2}{*}{$\begin{array}{c}\text { Design } \\
\text { Component }\end{array}$} & \multicolumn{6}{|c|}{ Current R Value } & \multirow{2}{*}{$\begin{array}{c}\text { deep } \\
\text { retrofit } \\
\text { R Value }\end{array}$} \\
\hline & T1 & $\mathrm{T} 2$ & T3 & $\mathrm{T} 4$ & $\mathrm{~T} 5$ & T6 & \\
\hline Attic & 8.91 & 28.16 & 28.79 & 22.43 & 26.18 & 30.15 & 60 \\
\hline $\begin{array}{c}\text { Foundation } \\
\text { Walls }\end{array}$ & 2.73 & 2.95 & 8.57 & 2.95 & 11.53 & 10.56 & 20 \\
\hline Walls & 9.71 & 10.62 & 10.16 & 9.60 & 14.71 & 14.88 & 40 \\
\hline Windows & 2 & 2 & 2 & 2 & 3 & 3 & 7 \\
\hline $\begin{array}{c}\text { Air Changes } \\
\text { Per Hour } \\
\text { (50p) }\end{array}$ & 10.88 & 5.72 & 7.22 & 5.1 & 5.52 & 3.31 & 0.6 \\
\hline
\end{tabular}

Typical $R$ values of windows have changed over time. Single-glazed windows with an $\mathrm{R}$ value of 1 (plus an outer storm window with $\mathrm{R}$ value of 1 ) were installed before the 1970s, double-glazed windows with an R value of 2 were installed from the 1970s to the 1980s, and double-glazed windows with a low-E coating and argon filled cavity (R value of 3) were installed after 1980s. In this study, it assumes that the $\mathrm{R}$ value of the windows of houses built before 1980 is 2 and the $\mathrm{R}$ value of the windows of houses built after 1980 is 3 . For window retrofits, all windows are replaced with 2 low-e coatings, argon filled triple-glazed windows with an $\mathrm{R}$ value of 7 (Table 3). In addition, the window category also includes doors, and the doors can be retrofitted from solid wood to 
steel with foam insulation so that they can achieve a similar upgrade to the windows. Therefore, this study includes window and door surface areas in a single category.

Table 3. The heat loss reduction from window improvement

\begin{tabular}{|c|c|c|c|c|c|c|}
\hline & T1 & T2 & T3 & T4 & T5 & T6 \\
\hline $\begin{array}{c}\text { R Value } \\
\text { before } \\
\text { retrofits }\end{array}$ & 2 & 2 & 2 & 2 & 3 & 3 \\
\hline $\begin{array}{c}\text { R Value after } \\
\text { retrofits (PH } \\
\text { Standard) }\end{array}$ & 7 & 7 & 7 & 7 & 7 & 7 \\
\hline $\begin{array}{c}\text { Heat Loss } \\
\text { Before } \\
\text { Retrofits (MJ) }\end{array}$ & 26,990 & 27,892 & 30,134 & 20,445 & 29,007 & 26,754 \\
\hline $\begin{array}{c}\text { Heat Loss } \\
\text { After } \\
\text { Retrofits (MJ) }\end{array}$ & 7,711 & 7,969 & 8,610 & 5,841 & 12,432 & 11,466 \\
\hline $\begin{array}{c}\text { Heat Loss } \\
\text { Reduction } \\
\text { (MJ) }\end{array}$ & 19,279 & 19,923 & 21,524 & 14,604 & 16,575 & 15,288 \\
\hline
\end{tabular}

For attic, foundation walls, and main walls retrofits, spray foam is often selected to be used as the insulation material. Spray foam is an excellent option inside wall cavities where it seals air leaks as well as adds high insulation values. The completed closed-cell spray polyurethane foam application provides an air and vapour barrier. Spray foam has a long lifetime and needs no replacement or replenishment. In this study, extruded polystyrene is selected to insulate the foundation floor because it is typically cheaper than spray foam. The foundation floor is rarely insulated when houses were built, so it assumes that there is no insulation in foundation floor of the six selected houses in this study. Given the $\mathrm{R}$ values per inch of closed-cell spray polyurethane foam and the extruded polystyrene: 6 and 5 respectively, the thickness of insulation materials required to insulate attic, foundation floor, foundation walls, and main walls can be calculated. In practice, the heat loss performance after the $\mathrm{R}$ value of foundation floor is raised to 10 and the $\mathrm{R}$ value of foundation walls raised to 20, is approximately equal because of the colder temperatures experienced outside the upper portion of the foundation wall. Finally, the heat loss reduction of each segment can be estimated according to the formula

$$
R=\triangle T / Q
$$

$\triangle \mathrm{T}$ represents temperature difference across the insulated segment (wall, floor, etc.) and Q represents the heat transfer per unit area per unit time (Table 4).

Table 4. The heat loss reduction after retrofitting main segments of typical houses

\begin{tabular}{|c|c|c|c|c|c|}
\hline & Insulation added & $\begin{array}{c}\text { Foundation } \\
\text { Floor }\end{array}$ & $\begin{array}{c}\text { Foundation } \\
\text { Walls }\end{array}$ & Attic & $\begin{array}{c}\text { Main } \\
\text { Walls }\end{array}$ \\
\hline \multirow{4}{*}{ T1 } & Thickness (inch) & 2 & 2.88 & 8.50 & 5.05 \\
\cline { 2 - 6 } & $\begin{array}{c}\text { Total amount } \\
\left(\mathrm{m}^{3}\right)\end{array}$ & 3.25 & 5.78 & 13.82 & 20.25 \\
\cline { 2 - 6 } & $\begin{array}{c}\text { Heat Loss Before } \\
\text { Retrofits (MJ) }\end{array}$ & \multicolumn{2}{|c|}{38,675} & 16,203 & 36,692 \\
\cline { 2 - 6 } & $\begin{array}{c}\text { Heat Loss After } \\
\text { Retrofits (MJ) }\end{array}$ & \multicolumn{2}{|c|}{5,279} & 2,406 & 8,907 \\
\cline { 2 - 6 } & $\begin{array}{c}\text { Heat Loss } \\
\text { Reduction (MJ) }\end{array}$ & \multicolumn{2}{|c|}{33,396} & 13,797 & 27,785 \\
\hline & Thickness (inch) & 2 & 2.84 & 5.31 & 4.90 \\
\hline
\end{tabular}

\begin{tabular}{|c|c|c|c|c|c|}
\hline \multirow[t]{4}{*}{$\mathrm{T} 2$} & $\begin{array}{c}\text { Total amount } \\
\left(\mathrm{m}^{3}\right)\end{array}$ & 4.57 & 6.76 & 12.14 & 11.67 \\
\hline & $\begin{array}{l}\text { Heat Loss Before } \\
\text { Retrofits (MJ) }\end{array}$ & \multicolumn{2}{|c|}{39,426} & 6,831 & 20,986 \\
\hline & $\begin{array}{c}\text { Heat Loss After } \\
\text { Retrofits (MJ) }\end{array}$ & \multicolumn{2}{|c|}{5,815} & 3,206 & 5,572 \\
\hline & $\begin{array}{c}\text { Heat Loss } \\
\text { Reduction(MJ) }\end{array}$ & \multicolumn{2}{|c|}{33,611} & 3,625 & 15,414 \\
\hline \multirow{5}{*}{ T3 } & Thickness (inch) & 2 & 1.88 & 5.20 & 4.97 \\
\hline & $\begin{array}{c}\text { Total amount } \\
\left(\mathrm{m}^{3}\right)\end{array}$ & 4.88 & 4.71 & 12.68 & 12.46 \\
\hline & $\begin{array}{l}\text { Heat Loss Before } \\
\text { Retrofits (MJ) }\end{array}$ & \multicolumn{2}{|c|}{26,137} & 6,285 & 17,608 \\
\hline & $\begin{array}{l}\text { Heat Loss After } \\
\text { Retrofits (MJ) }\end{array}$ & \multicolumn{2}{|c|}{11,120} & 3,016 & 4,472 \\
\hline & $\begin{array}{c}\text { Heat Loss } \\
\text { Reduction(MJ) }\end{array}$ & \multicolumn{2}{|c|}{14,937} & 3,269 & 13,136 \\
\hline \multirow{5}{*}{$\mathrm{T} 4$} & Thickness (inch) & 2 & 2.84 & 6.26 & 5.07 \\
\hline & $\begin{array}{c}\text { Total amount } \\
\left(\mathrm{m}^{3}\right)\end{array}$ & 4.88 & 7.12 & 15.26 & 12.71 \\
\hline & $\begin{array}{l}\text { Heat Loss Before } \\
\text { Retrofits (MJ) }\end{array}$ & \multicolumn{2}{|c|}{35,516} & 7,408 & 18,425 \\
\hline & $\begin{array}{c}\text { Heat Loss After } \\
\text { Retrofits (MJ) }\end{array}$ & \multicolumn{2}{|c|}{5,239} & 2,769 & 4,422 \\
\hline & $\begin{array}{c}\text { Heat Loss } \\
\text { Reduction(MJ) }\end{array}$ & \multicolumn{2}{|c|}{30,277} & 4,638 & 14,003 \\
\hline \multirow{5}{*}{ T5 } & Thickness (inch) & 2 & 1.42 & 5.64 & 4.22 \\
\hline & $\begin{array}{c}\text { Total amount } \\
\left(\mathrm{m}^{3}\right)\end{array}$ & 3.56 & 3.03 & 10.03 & 17.98 \\
\hline & $\begin{array}{c}\text { Heat Loss Before } \\
\text { Retrofits (MJ) }\end{array}$ & \multicolumn{2}{|c|}{21,958} & 5,769 & 24,863 \\
\hline & $\begin{array}{l}\text { Heat Loss After } \\
\text { Retrofits (MJ) }\end{array}$ & \multicolumn{2}{|c|}{12,659} & 2,517 & 9,143 \\
\hline & $\begin{array}{c}\text { Heat Loss } \\
\text { Reduction(MJ) }\end{array}$ & \multicolumn{2}{|c|}{9,299} & 3,252 & 15,720 \\
\hline \multirow{5}{*}{ T6 } & Thickness (inch) & 2 & 1.57 & 4.98 & 4.19 \\
\hline & $\begin{array}{c}\text { Total amount } \\
\left(\mathrm{m}^{3}\right)\end{array}$ & 3.56 & 3.35 & 8.85 & 17.86 \\
\hline & $\begin{array}{l}\text { Heat Loss Before } \\
\text { Retrofits (MJ) }\end{array}$ & \multicolumn{2}{|c|}{17,152} & 6,331 & 27,683 \\
\hline & $\begin{array}{l}\text { Heat Loss After } \\
\text { Retrofits (MJ) }\end{array}$ & \multicolumn{2}{|c|}{9,056} & 3,181 & 10,298 \\
\hline & $\begin{array}{c}\text { Heat Loss } \\
\text { Reduction(MJ) }\end{array}$ & \multicolumn{2}{|c|}{8,096} & 3,150 & 17,385 \\
\hline
\end{tabular}

Air leakage is defined as the flow of air through gaps and cracks in the building envelop. In order to reduce the heat loss from air flow, air sealing is necessary. For airsealing materials, weather stripping is a cost-effective way to reduce air leakage. In addition, caulking is used on the interior to seal small cracks and penetrations on the inside surface of walls and floors, and urethane foam is also good for filling larger joints and cavities. According to the $\mathrm{PH}$ air change standard, heat loss is proportional to air changes. However, the $\mathrm{PH}$ air change standard is very difficult to achieve in retrofit work, so this study assumes that a 50\% heat loss reduction in air leakage from that portion of the building envelop where retrofits are applied (Table 5). 
Table 5. The heat loss reduction from air tightness improvement

\begin{tabular}{|c|c|c|c|c|c|c|}
\hline & T1 & T2 & T3 & T4 & T5 & T6 \\
\hline $\begin{array}{c}\text { Air Changes } \\
\text { Per Hour } \\
\text { (50p) before } \\
\text { retrofits }\end{array}$ & 10.88 & 5.72 & 7.22 & 5.10 & 5.52 & 3.31 \\
\hline $\begin{array}{c}\text { Air Changes } \\
\text { Per Hour } \\
\text { (50p) (PH } \\
\text { Standard) }\end{array}$ & 0.6 & 0.6 & 0.6 & 0.6 & 0.6 & 0.6 \\
\hline $\begin{array}{c}\text { Heat Loss } \\
\text { Before } \\
\text { Retrofits(MJ) }\end{array}$ & 45,487 & 28,139 & 31,128 & 29,896 & 35,178 & 38,930 \\
\hline $\begin{array}{c}\text { Heat Loss } \\
\text { After } \\
\text { Retrofits(MJ) }\end{array}$ & 22,744 & 14,070 & 15,564 & 14,948 & 17,589 & 19,465 \\
\hline $\begin{array}{c}\text { Heat Loss } \\
\text { Reduction } \\
\text { (MJ) }\end{array}$ & 22,744 & 14,070 & 15,564 & 14,948 & 17,589 & 19,465 \\
\hline
\end{tabular}

Furthermore, in order to reduce natural gas consumption and $\mathrm{CO}_{2}$ emissions, a combination of solar water heater (effectively the pre-heater) and electric water heater will be used to replace natural gas for heating water. Therefore, approximately $50 \%$ of water heating needs can be met from solar, and the other $50 \%$ would be met from electricity $[7,8]$.

\section{Results and discussion}

As mentioned before, the natural gas consumed for heating water is replaced with solar energy and electricity which has low carbon intensity. Given an energy density of one cubic meter of natural gas approximately equals $38 \mathrm{MJ}$, the changes of heat loss of different segments are listed from table 6 to table 11. The $\mathrm{CO}_{2}$ emission reduction is estimated according to formula (2). $\mathrm{kgCO}_{2}=$ Electricity* $0.097 \mathrm{~kg} / \mathrm{kWh}+$ NaturalGas $* 1.88 \mathrm{~kg} / \mathrm{m}^{3}$ +Oil*2.83kg/L+Propane* $1.51 \mathrm{~kg} / \mathrm{L}$

Table 6. Comparison between before and after retrofit of a house built in $1945\left(169 \mathrm{~m}^{2}\right)$

\begin{tabular}{|c|c|c|c|}
\hline \multicolumn{2}{|c|}{ Fuel Type } & $\begin{array}{c}\text { Before Retrofit } \\
\text { Annual Consumption }\end{array}$ & $\begin{array}{c}\text { After Retrofit } \\
\text { Annual Consumption }\end{array}$ \\
\hline \multicolumn{2}{|c|}{ Electricity } & 9,389 KWh/yr & $13,500 \mathrm{KWh} / \mathrm{yr}$ \\
\hline \multirow[t]{2}{*}{$\begin{array}{l}\text { Natural } \\
\text { Gas }\end{array}$} & $\begin{array}{c}\text { Space } \\
\text { Heating }\end{array}$ & $4,533 \mathrm{~m}^{3} / \mathrm{yr}(78 \%)$ & $1,454 \mathrm{~m}^{3} / \mathrm{yr}(96 \%)$ \\
\hline & $\begin{array}{l}\text { Water } \\
\text { Heating }\end{array}$ & $779 \mathrm{~m}^{3} / \mathrm{yr}(55 \%)$ & 0 \\
\hline \multicolumn{2}{|c|}{ Solar Energy } & 0 & $14,801 \mathrm{MJ} / \mathrm{yr}$ \\
\hline \multicolumn{2}{|c|}{ Attic Heat Loss } & $16,203 \mathrm{MJ}$ & $2,406 \mathrm{MJ}$ \\
\hline \multicolumn{2}{|c|}{ Attic Heat Loss Savings } & \multicolumn{2}{|c|}{$85 \%$} \\
\hline \multicolumn{2}{|c|}{ Foundation Heat Loss } & $38,675 \mathrm{MJ}$ & $5,279 \mathrm{MJ}$ \\
\hline \multicolumn{2}{|c|}{$\begin{array}{l}\text { Foundation Heat Loss } \\
\text { Savings }\end{array}$} & \multicolumn{2}{|c|}{$86 \%$} \\
\hline \multicolumn{2}{|c|}{ Walls Heat Loss } & 36,692 MJ & $8,907 \mathrm{MJ}$ \\
\hline \multicolumn{2}{|c|}{ Walls Heat Loss Savings } & \multicolumn{2}{|c|}{$76 \%$} \\
\hline \multicolumn{2}{|c|}{$\begin{array}{c}\text { Windows/Doors Heat } \\
\text { Loss }\end{array}$} & $26,990 \mathrm{MJ}$ & $7,711 \mathrm{MJ}$ \\
\hline \multicolumn{2}{|c|}{$\begin{array}{l}\text { Windows/Doors Heat } \\
\text { Loss Savings }\end{array}$} & \multicolumn{2}{|c|}{$71 \%$} \\
\hline \multicolumn{2}{|c|}{ Air Leaks Heat Loss } & $45,487 \mathrm{MJ}$ & $22,744 \mathrm{MJ}$ \\
\hline \multicolumn{2}{|c|}{$\begin{array}{c}\text { Air Leaks Heat Loss } \\
\text { Savings }\end{array}$} & \multicolumn{2}{|c|}{$50 \%$} \\
\hline \multicolumn{2}{|c|}{$\begin{array}{l}\text { Space Heating Energy } \\
\text { (Natural Gas) }\end{array}$} & $172,264 \mathrm{MJ} / \mathrm{yr}$ & $\begin{array}{c}55,263 \mathrm{MJ} / \mathrm{yr}(> \\
\left.50 \mathrm{kWh} / \mathrm{m}^{2} \mathrm{a}\right)\end{array}$ \\
\hline \multicolumn{2}{|c|}{$\begin{array}{c}\text { Total Energy } \\
\text { (Electricity+Natural Gas) }\end{array}$} & $231,708 \mathrm{MJ} / \mathrm{yr}$ & $\begin{array}{c}103,863 \mathrm{MJ} / \mathrm{yr}(> \\
\left.120 \mathrm{kWh} / \mathrm{m}^{2} \mathrm{a}\right)\end{array}$ \\
\hline \multicolumn{2}{|c|}{ Total Energy Savings } & \multicolumn{2}{|c|}{$55 \%$} \\
\hline \multicolumn{2}{|c|}{$\mathrm{CO}_{2}$ Emissions Reduction } & \multicolumn{2}{|c|}{$6,854 \mathrm{~kg} / \mathrm{yr}$} \\
\hline
\end{tabular}

Table 7. Comparison between before and after retrofit of a house built in $1959\left(191 \mathrm{~m}^{2}\right)$

\begin{tabular}{|c|c|c|c|}
\hline \multicolumn{2}{|c|}{ Fuel Type } & $\begin{array}{c}\text { Before Retrofit } \\
\text { Annual Consumption }\end{array}$ & $\begin{array}{l}\text { After Retrofit } \\
\text { Annual } \\
\text { Consumption }\end{array}$ \\
\hline \multicolumn{2}{|c|}{ Electricity } & $10,760 \mathrm{KWh} / \mathrm{yr}$ & 14,106 KWh/yr \\
\hline \multirow[t]{2}{*}{$\begin{array}{c}\text { Natural } \\
\text { Gas }\end{array}$} & $\begin{array}{c}\text { Space } \\
\text { Heating } \\
\end{array}$ & $\begin{array}{ll} & 3,256 \mathrm{~m}^{3} / \mathrm{yr} \\
& \\
\end{array}$ & $975 \mathrm{~m}^{3} / \mathrm{yr}(96 \%)$ \\
\hline & $\begin{array}{c}\text { Water } \\
\text { Heating }\end{array}$ & $634 \mathrm{~m}^{3} / \mathrm{yr}(55 \%)$ & 0 \\
\hline \multicolumn{2}{|c|}{ Solar Energy } & 0 & $12,046 \mathrm{MJ} / \mathrm{yr}$ \\
\hline \multicolumn{2}{|c|}{ Attic Heat Loss } & 6,831 MJ & 3,206 MJ \\
\hline \multicolumn{2}{|c|}{ Attic Heat Loss Savings } & \multicolumn{2}{|c|}{$53 \%$} \\
\hline Founda & Heat Loss & $39,426 \mathrm{MJ}$ & 5,815 MJ \\
\hline \multicolumn{2}{|c|}{$\begin{array}{c}\text { Foundation Heat Loss } \\
\text { Savings }\end{array}$} & \multicolumn{2}{|c|}{$85 \%$} \\
\hline \multicolumn{2}{|c|}{ Walls Heat Loss } & $20,986 \mathrm{MJ}$ & 5,572 MJ \\
\hline \multicolumn{2}{|c|}{ Walls Heat Loss Savings } & \multicolumn{2}{|c|}{$73 \%$} \\
\hline \multicolumn{2}{|c|}{$\begin{array}{c}\text { Windows/Doors Heat } \\
\text { Loss } \\
\end{array}$} & $27,892 \mathrm{MJ}$ & 7,969 MJ \\
\hline \multicolumn{2}{|c|}{$\begin{array}{c}\text { Windows/Doors Heat } \\
\text { Loss Savings } \\
\end{array}$} & \multicolumn{2}{|c|}{$71 \%$} \\
\hline \multirow{2}{*}{\multicolumn{2}{|c|}{$\begin{array}{c}\text { Air Leaks Heat Loss } \\
\text { Air Leaks Heat Loss } \\
\text { Savings }\end{array}$}} & $28,139 \mathrm{MJ}$ & $14,070 \mathrm{MJ}$ \\
\hline & & \multicolumn{2}{|c|}{$50 \%$} \\
\hline \multicolumn{2}{|c|}{$\begin{array}{l}\text { Space Heating Energy } \\
\text { (Natural Gas) }\end{array}$} & $123,710 \mathrm{MJ} / \mathrm{yr}$ & $\begin{array}{c}37,067 \mathrm{MJ} / \mathrm{yr}(> \\
\left.50 \mathrm{kWh} / \mathrm{m}^{2} \mathrm{a}\right)\end{array}$ \\
\hline \multicolumn{2}{|c|}{$\begin{array}{c}\text { Total Energy } \\
\text { (Electricity+Natural Gas) }\end{array}$} & $183,665 \mathrm{MJ} / \mathrm{yr}$ & $\begin{array}{c}87,849 \mathrm{MJ} / \mathrm{yr}(> \\
\left.120 \mathrm{kWh} / \mathrm{m}^{2} \mathrm{a}\right)\end{array}$ \\
\hline \multicolumn{2}{|c|}{ Total Energy Savings } & \multicolumn{2}{|c|}{$52 \%$} \\
\hline \multicolumn{2}{|c|}{$\mathrm{CO}_{2}$ Emissions Reduction } & \multicolumn{2}{|c|}{$5,805 \mathrm{~kg} / \mathrm{yr}$} \\
\hline
\end{tabular}

Table 8. Comparison between before and after retrofit of a house built in $1966\left(204 \mathrm{~m}^{2}\right)$

\begin{tabular}{|c|c|c|c|}
\hline \multicolumn{2}{|c|}{ Fuel Type } & $\begin{array}{c}\text { Before Retrofit } \\
\text { Annual Consumption }\end{array}$ & $\begin{array}{c}\text { After Retrofit } \\
\text { Annual } \\
\text { Consumption }\end{array}$ \\
\hline \multicolumn{2}{|c|}{ Electricity } & 10,818 KWh/yr & $14,148 \mathrm{KWh} / \mathrm{yr}$ \\
\hline \multirow[t]{2}{*}{$\begin{array}{l}\text { Natural } \\
\text { Gas }\end{array}$} & $\begin{array}{c}\text { Space } \\
\text { Heating }\end{array}$ & $\begin{array}{ll} & 3,082 \mathrm{~m}^{3} / \mathrm{yr} \\
(78 \%) & \\
\end{array}$ & $1,281 \mathrm{~m}^{3} / \mathrm{yr}(96 \%)$ \\
\hline & $\begin{array}{c}\text { Water } \\
\text { Heating }\end{array}$ & $631 \mathrm{~m}^{3} / \mathrm{yr}(55 \%)$ & 0 \\
\hline \multicolumn{2}{|c|}{ Solar Energy } & 0 & $11,989 \mathrm{MJ} / \mathrm{yr}$ \\
\hline \multicolumn{2}{|c|}{ Attic Heat Loss } & $6,285 \mathrm{MJ}$ & $3,016 \mathrm{MJ}$ \\
\hline \multicolumn{2}{|c|}{ Attic Heat Loss Savings } & \multicolumn{2}{|c|}{$52 \%$} \\
\hline \multicolumn{2}{|c|}{ Foundation Heat Loss } & $26,137 \mathrm{MJ}$ & $11,120 \mathrm{MJ}$ \\
\hline \multicolumn{2}{|c|}{$\begin{array}{c}\text { Foundation Heat Loss } \\
\text { Savings }\end{array}$} & \multicolumn{2}{|c|}{$57 \%$} \\
\hline \multicolumn{2}{|c|}{ Walls Heat Loss } & $17,608 \mathrm{MJ}$ & 4,472 MJ \\
\hline \multicolumn{2}{|c|}{ Walls Heat Loss Savings } & \multicolumn{2}{|c|}{$75 \%$} \\
\hline \multicolumn{2}{|c|}{$\begin{array}{c}\text { Windows/Doors Heat } \\
\text { Loss } \\
\end{array}$} & $30,143 \mathrm{MJ}$ & $8,610 \mathrm{MJ}$ \\
\hline \multicolumn{2}{|c|}{$\begin{array}{l}\text { Windows/Doors Heat } \\
\text { Loss Savings }\end{array}$} & \multicolumn{2}{|c|}{$71 \%$} \\
\hline \multicolumn{2}{|c|}{ Air Leaks Heat Loss } & $31,128 \mathrm{MJ}$ & $15,564 \mathrm{MJ}$ \\
\hline \multicolumn{2}{|c|}{$\begin{array}{c}\text { Air Leaks Heat Loss } \\
\text { Savings }\end{array}$} & \multicolumn{2}{|c|}{$50 \%$} \\
\hline \multicolumn{2}{|c|}{$\begin{array}{c}\text { Space Heating Energy } \\
\text { (Natural Gas) }\end{array}$} & $117,114 \mathrm{MJ} / \mathrm{yr}$ & $\begin{array}{c}48,684 \mathrm{MJ} / \mathrm{yr}(> \\
\left.50 \mathrm{kWh} / \mathrm{m}^{2} \mathrm{a}\right)\end{array}$ \\
\hline \multicolumn{2}{|c|}{$\begin{array}{c}\text { Total Energy } \\
\text { (Electricity+Natural Gas) }\end{array}$} & $177,304 \mathrm{MJ} / \mathrm{yr}$ & $\begin{array}{c}99,617 \mathrm{MJ} / \mathrm{yr}(> \\
\left.120 \mathrm{kWh} / \mathrm{m}^{2} \mathrm{a}\right)\end{array}$ \\
\hline \multicolumn{2}{|c|}{ Total Energy Savings } & \multicolumn{2}{|c|}{$44 \%$} \\
\hline \multicolumn{2}{|c|}{$\mathrm{CO}_{2}$ Emissions Reduction } & \multicolumn{2}{|c|}{$4,249 \mathrm{~kg} / \mathrm{yr}$} \\
\hline
\end{tabular}


Table 9. Comparison between before and after retrofit of a house built in $1975\left(166 \mathrm{~m}^{2}\right)$

\begin{tabular}{|c|c|c|c|}
\hline \multicolumn{2}{|c|}{ Fuel Type } & $\begin{array}{c}\text { Before Retrofit } \\
\text { Annual Consumption }\end{array}$ & $\begin{array}{l}\text { After Retrofit } \\
\text { Annual } \\
\text { Consumption } \\
\end{array}$ \\
\hline \multicolumn{2}{|c|}{ Electricity } & 9,600 KWh/yr & $13,643 \mathrm{KWh} / \mathrm{yr}$ \\
\hline \multirow[t]{2}{*}{$\begin{array}{c}\text { Natural } \\
\text { Gas }\end{array}$} & $\begin{array}{c}\text { Space } \\
\text { Heating } \\
\end{array}$ & $\begin{array}{ll}(78 \%) & 2,871 \mathrm{~m}^{3} / \mathrm{yr} \\
\end{array}$ & $806 \mathrm{~m}^{3} / \mathrm{yr}(96 \%)$ \\
\hline & $\begin{array}{c}\text { Water } \\
\text { Heating } \\
\end{array}$ & $766 \mathrm{~m}^{3} / \mathrm{yr}(55 \%)$ & 0 \\
\hline \multicolumn{2}{|c|}{ Solar Energy } & 0 & $14,554 \mathrm{MJ} / \mathrm{yr}$ \\
\hline \multicolumn{2}{|c|}{ Attic Heat Loss } & 7,408 MJ & $2,769 \mathrm{MJ}$ \\
\hline \multicolumn{2}{|c|}{ Attic Heat Loss Savings } & \multicolumn{2}{|c|}{$63 \%$} \\
\hline \multicolumn{2}{|c|}{ Foundation Heat Loss } & $35,516 \mathrm{MJ}$ & $5,239 \mathrm{MJ}$ \\
\hline \multicolumn{2}{|c|}{$\begin{array}{l}\text { Foundation Heat Loss } \\
\text { Savings }\end{array}$} & \multicolumn{2}{|c|}{$85 \%$} \\
\hline \multicolumn{2}{|c|}{ Walls Heat Loss } & $18,425 \mathrm{MJ}$ & $4,422 \mathrm{MJ}$ \\
\hline \multicolumn{2}{|c|}{ Walls Heat Loss Savings } & \multicolumn{2}{|c|}{$76 \%$} \\
\hline \multicolumn{2}{|c|}{$\begin{array}{c}\text { Windows/Doors Heat } \\
\text { Loss }\end{array}$} & $20,445 \mathrm{MJ}$ & $5,841 \mathrm{MJ}$ \\
\hline \multicolumn{2}{|c|}{$\begin{array}{l}\text { Windows/Doors Heat } \\
\text { Loss Savings }\end{array}$} & \multicolumn{2}{|c|}{$71 \%$} \\
\hline \multirow{2}{*}{\multicolumn{2}{|c|}{$\begin{array}{l}\text { Air Leaks Heat Loss } \\
\text { Air Leaks Heat Loss } \\
\text { Savings }\end{array}$}} & 29,896 MJ & $14,948 \mathrm{MJ}$ \\
\hline & & & \\
\hline \multicolumn{2}{|c|}{$\begin{array}{l}\text { Space Heating Energy } \\
\text { (Natural Gas) }\end{array}$} & $109,094 \mathrm{MJ} / \mathrm{yr}$ & $\begin{array}{c}30,624 \mathrm{MJ} / \mathrm{yr}(> \\
\left.50 \mathrm{kWh} / \mathrm{m}^{2} \mathrm{a}\right)\end{array}$ \\
\hline \multicolumn{2}{|c|}{$\begin{array}{c}\text { Total Energy } \\
\text { (Electricity+Natural Gas) }\end{array}$} & $170,087 \mathrm{MJ} / \mathrm{yr}$ & $\begin{array}{c}79739 \mathrm{MJ} / \mathrm{yr}(> \\
\left.120 \mathrm{kWh} / \mathrm{m}^{2} \mathrm{a}\right)\end{array}$ \\
\hline \multicolumn{2}{|c|}{ Total Energy Savings } & \multicolumn{2}{|c|}{$53 \%$} \\
\hline \multicolumn{2}{|c|}{$\mathrm{CO}_{2}$ Emissions Reduction } & \multicolumn{2}{|c|}{$4,930 \mathrm{~kg} / \mathrm{yr}$} \\
\hline
\end{tabular}

Table 10. Comparison between before and after retrofit of a house built in $1986\left(207 \mathrm{~m}^{2}\right)$

\begin{tabular}{|c|c|c|c|}
\hline \multicolumn{2}{|c|}{ Fuel Type } & $\begin{array}{c}\text { Before Retrofit } \\
\text { Annual Consumption }\end{array}$ & $\begin{array}{c}\text { After Retrofit } \\
\text { Annual } \\
\text { Consumption }\end{array}$ \\
\hline \multicolumn{2}{|c|}{ Electricity } & 9,433 KWh/yr & 13,471 KWh/yr \\
\hline \multirow[t]{2}{*}{$\begin{array}{l}\text { Natural } \\
\text { Gas }\end{array}$} & $\begin{array}{c}\text { Space } \\
\text { Heating }\end{array}$ & (78\%) & $1,167 \mathrm{~m}^{3} / \mathrm{yr}(96 \%)$ \\
\hline & $\begin{array}{l}\text { Water } \\
\text { Heating }\end{array}$ & $765 \mathrm{~m}^{3} / \mathrm{yr}(55 \%)$ & 0 \\
\hline \multicolumn{2}{|c|}{ Solar Energy } & 0 & $14,535 \mathrm{MJ} / \mathrm{yr}$ \\
\hline \multicolumn{2}{|c|}{ Attic Heat Loss } & $5,769 \mathrm{MJ}$ & $2,517 \mathrm{MJ}$ \\
\hline \multicolumn{2}{|c|}{ Attic Heat Loss Savings } & \multicolumn{2}{|c|}{$56 \%$} \\
\hline Found & Heat Loss & $21,958 \mathrm{MJ}$ & $12,659 \mathrm{MJ}$ \\
\hline \multicolumn{2}{|c|}{$\begin{array}{c}\text { Foundation Heat Loss } \\
\text { Savings }\end{array}$} & \multicolumn{2}{|c|}{$42 \%$} \\
\hline $\mathrm{Wal}$ & at Loss & $24,863 \mathrm{MJ}$ & 9,143 MJ \\
\hline \multirow{2}{*}{\multicolumn{2}{|c|}{$\begin{array}{c}\text { Walls Heat Loss Savings } \\
\text { Windows/Doors Heat } \\
\text { Loss }\end{array}$}} & \multicolumn{2}{|c|}{$63 \%$} \\
\hline & & $29,007 \mathrm{MJ}$ & $12,432 \mathrm{MJ}$ \\
\hline \multicolumn{2}{|c|}{$\begin{array}{l}\text { Windows/Doors Heat } \\
\text { Loss Savings }\end{array}$} & \multicolumn{2}{|c|}{$57 \%$} \\
\hline \multicolumn{2}{|c|}{ Air Leaks Heat Loss } & $35,178 \mathrm{MJ}$ & 17,589 MJ \\
\hline \multicolumn{2}{|c|}{$\begin{array}{c}\text { Air Leaks Heat Loss } \\
\text { Savings }\end{array}$} & \multicolumn{2}{|c|}{$50 \%$} \\
\hline \multicolumn{2}{|c|}{$\begin{array}{l}\text { Space Heating Energy } \\
\text { (Natural Gas) }\end{array}$} & $106,778 \mathrm{MJ} / \mathrm{yr}$ & $\begin{array}{c}44,343 \mathrm{MJ} / \mathrm{yr}(> \\
\left.50 \mathrm{kWh} / \mathrm{m}^{2} \mathrm{a}\right)\end{array}$ \\
\hline \multicolumn{2}{|c|}{$\begin{array}{c}\text { Total Energy } \\
\text { (Electricity+Natural Gas) }\end{array}$} & $167,153 \mathrm{MJ} / \mathrm{yr}$ & $\begin{array}{c}92,838 \mathrm{MJ} / \mathrm{yr}(> \\
\left.120 \mathrm{kWh} / \mathrm{m}^{2} \mathrm{a}\right)\end{array}$ \\
\hline \multicolumn{2}{|c|}{ Total Energy Savings } & \multicolumn{2}{|c|}{$44 \%$} \\
\hline \multicolumn{2}{|c|}{$\mathrm{CO}_{2}$ Emissions Reduction } & \multicolumn{2}{|c|}{$4,135 \mathrm{~kg} / \mathrm{yr}$} \\
\hline
\end{tabular}

Table 11. Comparison between before and after retrofit of a house built in $1995\left(278 \mathrm{~m}^{2}\right)$

\begin{tabular}{|c|c|c|c|}
\hline \multicolumn{2}{|c|}{ Fuel Type } & $\begin{array}{c}\text { Before Retrofit } \\
\text { Annual Consumption }\end{array}$ & $\begin{array}{c}\text { After Retrofit } \\
\text { Annual } \\
\text { Consumption }\end{array}$ \\
\hline \multicolumn{2}{|c|}{ Electricity } & $10,824 \mathrm{KWh} / \mathrm{yr}$ & $13,922 \mathrm{KWh} / \mathrm{yr}$ \\
\hline \multirow{2}{*}{$\begin{array}{c}\text { Natural } \\
\text { Gas }\end{array}$} & $\begin{array}{c}\text { Space } \\
\text { Heating }\end{array}$ & $2,247 \mathrm{~m}^{3} / \mathrm{yr}$ & $579 \mathrm{~m}^{3} / \mathrm{yr}(96 \%)$ \\
\cline { 2 - 4 } & $\begin{array}{c}\text { Water } \\
\text { Heating }\end{array}$ & $587 \mathrm{~m}^{3} / \mathrm{yr}(55 \%)$ & 0 \\
\hline \multicolumn{2}{|c|}{ Solar Energy } & 0 & $11,153 \mathrm{MJ} / \mathrm{yr}$ \\
\hline
\end{tabular}

\begin{tabular}{|c|c|c|}
\hline Attic Heat Loss & 6,331 MJ & 3,181 MJ \\
\hline Attic Heat Loss Savings & \multicolumn{2}{|c|}{$50 \%$} \\
\hline Foundation Heat Loss & 17,152 MJ & $9,056 \mathrm{MJ}$ \\
\hline $\begin{array}{c}\text { Foundation Heat Loss } \\
\text { Savings } \\
\end{array}$ & \multicolumn{2}{|c|}{$47 \%$} \\
\hline Walls Heat Loss & $27,683 \mathrm{MJ}$ & $10,298 \mathrm{MJ}$ \\
\hline Walls Heat Loss Savings & \multicolumn{2}{|c|}{$63 \%$} \\
\hline $\begin{array}{c}\text { Windows/Doors Heat } \\
\text { Loss }\end{array}$ & $26,754 \mathrm{MJ}$ & $11,466 \mathrm{MJ}$ \\
\hline $\begin{array}{c}\text { Windows/Doors Heat } \\
\text { Loss Savings } \\
\end{array}$ & \multicolumn{2}{|c|}{$57 \%$} \\
\hline Air Leaks Heat Loss & $38,930 \mathrm{MJ}$ & $19,465 \mathrm{MJ}$ \\
\hline $\begin{array}{l}\text { Air Leaks Heat Loss } \\
\text { Savings }\end{array}$ & \multicolumn{2}{|c|}{$50 \%$} \\
\hline $\begin{array}{l}\text { Space Heating Energy } \\
\text { (Natural Gas) }\end{array}$ & $85,368 \mathrm{MJ} / \mathrm{yr}$ & $\begin{array}{c}21,984 \mathrm{MJ} / \mathrm{yr}(< \\
\left.50 \mathrm{kWh} / \mathrm{m}^{2} \mathrm{a}\right)\end{array}$ \\
\hline $\begin{array}{c}\text { Total Energy } \\
\text { (Electricity+Natural Gas) }\end{array}$ & $144,548 \mathrm{MJ} / \mathrm{yr}$ & $\begin{array}{r}72,103 \mathrm{MJ} / \mathrm{yr}(< \\
\left.120 \mathrm{kWh} / \mathrm{m}^{2} \mathrm{a}\right)\end{array}$ \\
\hline Total Energy Savings & \multicolumn{2}{|c|}{$50 \%$} \\
\hline $\mathrm{CO}_{2}$ Emissions Reduction & \multicolumn{2}{|c|}{$3,939 \mathrm{~kg} / \mathrm{yr}$} \\
\hline
\end{tabular}

Tables 6-11 summarise the heat loss reduction in attic, foundation, walls, windows/doors and air leaks after deep retrofits are undertaken. The primary $\mathrm{PH}$ standard is that the maximum annual space heating energy consumption is no more than $15 \mathrm{kWh} / \mathrm{m}^{2} \mathrm{a}$. However, the northern European target of $50 \mathrm{kWh} / \mathrm{m}^{2}$ a would be more feasible for Canadian retrofits because of more similar weather conditions. Therefore, the retrofit goals in this study are that the maximum annual space heating energy consumption is $50 \mathrm{kWh} / \mathrm{m}^{2}$ a instead of $15 \mathrm{kWh} / \mathrm{m}^{2}$ a and the total maximum annual energy consumption is 120 $\mathrm{kWh} / \mathrm{m}^{2} \mathrm{a}$.

According to above analysis, it can be concluded that only the sixth typical house built in 1995 achieves the PH standard. Its annual space heating energy consumption changes from $86 \mathrm{kWh} / \mathrm{m}^{2}$ a to $22 \mathrm{kWh} / \mathrm{m}^{2}$ a which is less than the PH standard of $50 \mathrm{kWh} / \mathrm{m}^{2} \mathrm{a}$, and its total annual energy consumption changes from $144 \mathrm{kWh} / \mathrm{m}^{2}$ a to 87 $\mathrm{kWh} / \mathrm{m}^{2} \mathrm{a}$ which is less than the PH standard of 120 $\mathrm{kWh} / \mathrm{m}^{2} \mathrm{a}$ after retrofits. The annual space heating energy consumption of the first typical house built in 1945 changes from $283 \mathrm{kWh} / \mathrm{m}^{2}$ a to $91 \mathrm{kWh} / \mathrm{m}^{2} \mathrm{a}$, and its total annual energy consumption changes from $381 \mathrm{kWh} / \mathrm{m}^{2} \mathrm{a}$ to $171 \mathrm{kWh} / \mathrm{m}^{2} \mathrm{a}$ after retrofits. The annual space heating energy consumption of the second typical house built in 1959 changes from $178 \mathrm{kWh} / \mathrm{m}^{2}$ a to $54 \mathrm{kWh} / \mathrm{m}^{2}$ a, and its total annual energy consumption changes from 267 $\mathrm{kWh} / \mathrm{m}^{2}$ a to $128 \mathrm{kWh} / \mathrm{m}^{2}$ a after retrofits. The annual space heating energy consumption of the third typical house built in 1966 changes from $159 \mathrm{kWh} / \mathrm{m}^{2}$ a to 66 $\mathrm{kWh} / \mathrm{m}^{2} \mathrm{a}$, and its total annual energy consumption changes from $241 \mathrm{kWh} / \mathrm{m}^{2} \mathrm{a}$ to $136 \mathrm{kWh} / \mathrm{m}^{2} \mathrm{a}$ after retrofits. The annual space heating energy consumption of the fourth typical house built in 1975 changes from $183 \mathrm{kWh} / \mathrm{m}^{2}$ a to $52 \mathrm{kWh} / \mathrm{m}^{2} \mathrm{a}$, and its total annual energy consumption changes from $285 \mathrm{kWh} / \mathrm{m}^{2} \mathrm{a}$ to 133 $\mathrm{kWh} / \mathrm{m}^{2} \mathrm{a}$ after retrofits. The annual space heating energy consumption of the fifth typical house built in 1986 changes from $143 \mathrm{kWh} / \mathrm{m}^{2} \mathrm{a}$ to $59 \mathrm{kWh} / \mathrm{m}^{2} \mathrm{a}$, and its total annual energy consumption changes from $224 \mathrm{kWh} / \mathrm{m}^{2} \mathrm{a}$ to $125 \mathrm{kWh} / \mathrm{m}^{2}$ a after retrofits. Therefore, the first five typical houses do not achieve the PH standard after the proposed deep retrofits.

Since the energy consumption of the typical house represents the average level of energy consumption of 
corresponding house category, the total amount of energy savings and $\mathrm{CO}_{2}$ emissions reduction of each house category can be calculated by multiplying the number of houses in this category with the amount of energy savings and $\mathrm{CO}_{2}$ emissions reduction of corresponding typical house (Table 12).

Table 12. Energy savings and $\mathrm{CO}_{2}$ emissions reduction after retrofits

\begin{tabular}{|c|c|c|c|}
\hline $\begin{array}{c}\text { House } \\
\text { Category }\end{array}$ & Number of Houses & $\begin{array}{c}\text { Energy savings } \\
\left(* 10^{9} \mathrm{MJ} / \mathrm{yr}\right)\end{array}$ & $\begin{array}{c}\mathrm{CO}_{2} \text { Emissions } \\
\text { Reduction } \\
\left(* 10^{8} \mathrm{~kg} / \mathrm{yr}\right)\end{array}$ \\
\hline C1 & 21,665 & 2.77 & 1.48 \\
\hline C2 & 21,080 & 2.02 & 1.22 \\
\hline C3 & 27,090 & 2.10 & 1.15 \\
\hline C4 & 32,845 & 2.97 & 1.62 \\
\hline C5 & 31,450 & 2.34 & 1.30 \\
\hline C6 & 26,995 & 1.96 & 1.06 \\
\hline Total & 161,125 & 14.16 & 7.83 \\
\hline
\end{tabular}

As shown in Table 12, the total amounts of potential annual energy savings and $\mathrm{CO}_{2}$ emissions reduction are $14.16 \times 10^{9} \mathrm{MJ} / \mathrm{yr}$ and $7.83 \times 10^{8} \mathrm{~kg} / \mathrm{yr}$ if deep retrofits are made to all houses in Waterloo Region.

\section{Conclusion}

This study shows the potential retrofit benefits to the public by estimating the energy savings and $\mathrm{CO}_{2}$ emissions reduction from retrofitted houses of Waterloo Region. This paper estimates that the total potential annual energy savings and $\mathrm{CO}_{2}$ emissions reductions are $14.16 \times 109 \mathrm{MJ} / \mathrm{yr}$ and $7.83 \times 108 \mathrm{~kg} / \mathrm{yr}$ if Waterloo Region adopts deep retrofit standards to move the residential sector toward PH levels of energy performance.

According to the calculations, it can be concluded that after implementing the deep retrofit scenario, only the house built in 1995 achieves the PH standard with annual space heating energy consumption of $37 \mathrm{kWh} / \mathrm{m}^{2} \mathrm{a}$ and annual energy consumption of $87 \mathrm{kWh} / \mathrm{m}^{2} \mathrm{a}$. In addition, the energy consumption and $\mathrm{CO}_{2}$ emissions of the house built in 1945 after retrofits are reduced by 55\% and 63\%. The energy consumption and $\mathrm{CO}_{2}$ emissions of the house built in 1959 after retrofits are reduced by 52\% and 69\%. The energy consumption and $\mathrm{CO}_{2}$ emissions of the house built in 1966 after retrofits are reduced by 44\% and 53\%. The energy consumption and $\mathrm{CO}_{2}$ emissions of the house built in1975 after retrofits are reduced by $53 \%$ and $63 \%$. The energy consumption and $\mathrm{CO}_{2}$ emissions of the house built in 1986 after retrofits are reduced by 44\% and 54\%. Therefore, these five typical houses do not achieve the $\mathrm{PH}$ standard after retrofits, and the results indicate that in order to achieve the $\mathrm{PH}$ energy intensity standard, even deeper retrofits would be required. In practice, the $\mathrm{PH}$ energy intensity standard is difficult to achieve in retrofit projects. Firstly, the European $\mathrm{PH}$ standard is not appropriate for retrofitting houses in Canada because the climates are not comparable. Canada has colder design heating loads than German locations which means that the insulation required to meet the German energy budget are thicker in Canada. Secondly, the PH standard is based on modeled performance instead of measured performance. It is difficult to be widely applied in actual work.

This paper demonstrates the potential benefits of deep residential retrofits to homeowners, researchers, and governments. It will also be helpful for future residential energy research and making environmentally friendly policies and regulations.

\section{References}

[1] CMHC. (2007). CHS Demography 2006. Canada, Canada: Canada Mortgage and Housing Corporation. http://www.cmhcschl.gc.ca/odpub/pdf/65704.pdf?lang=en

[2] Climate Action Plan for Waterloo Region: Living Smarter in 2020, http://www.climateactionwr.ca/learnmore/documents/

[3] The Regional Municipality of Waterloo. (2013). Waterloo Region: A Housing Overview. Kitchener, Ontario.

http://communityservices.regionofwaterloo.ca/en/ho using/resources/2013 Waterloo Region A Housing Overview.pdf

[4] Wimmers, G. (2011). Passive house in cold climates. Retrieved December 19, 2012, from http://www.cagbc.org/AM/PDF/Passive\%20House\% 20in\%20cold\%20climates.pdf

[5] Parker, P., Scott, D., Rowlands, I. H. 2000. Residential energy consumption and GHG emissions: Global context for local action. Environments. 28(3) 11-28.

[6] Statistics Canada http://www.statcan.gc.ca/tablestableaux/sum-som/101/cst01/famil125a-eng.htm

[7] NRCan. (2013a). Energy Efficiency Update 2013 Energy Efficiency: Taking a Balanced Approach. Energy and Mines Ministers' Conference Yellowknife, Northwest Territories August 2013. Retrieved from http://www.nrcan.gc.ca/sites/www.nrcan.gc.ca/files/ pdf/publications/emmc/EE_update2013_e.pdf

[8] NRCan. (2013b). About the R-2000 Standard. Retrieved from https://www.nrcan.gc.ca/energy/efficiency/housing/n ew-homes/r-2000/5095 